\title{
The Local Group census: IC-10
}

\author{
Shelley A. Wright, Robert Greimel, Daniel J. Lennon, and \\ Romano L.M. Corradi \\ The Isaac Newton Group of Telescopes, Apartado de Correos 321, \\ E-38700, Santa Cruz de La Palma, Canary Islands, España
}

\author{
Nicholas A. Walton \\ Institute of Astronomy, Madingley Road, Cambridge, CB3 OHA, UK
}

\begin{abstract}
We have begun a census of various stellar groups in Local Group Galaxies, using the wide Field Camera on the Isaac Newton Telescope on La Palma. Here we present a preliminary color-magnitude diagram for the dwarf irregular galaxy IC-10. At present time, metallicity of IC-10 is measured to be $Z=0.005$ (Garnett 1990). Comparison with recent literature values of reddening and distance suggest that IC-10's distance is $\sim 1 \mathrm{Mpc}$. Our comprehensive widefield survey encompasses both broad $\left(g^{\prime}, r^{\prime}, i^{\prime}\right)$ and narrow-band (O III, He II, $\mathrm{H} \alpha$, S II, Strömgren $y$ ) observations to look for emission-line objects, including Wolf-Rayet stars and Luminous Blue Variables. The analysis also yields the coordinates of massive stars to an accuracy sufficient for follow-up multi-object spectroscopic observations.
\end{abstract}

\section{Local Group census and preliminary results for IC-10}

The Local Group Census (LGC) is conducted at the Isaac Newton Group of Telescopes, La Palma, with the wide Field Camera (WFC) on the $2.5 \mathrm{~m}$ Isaac Newton Telescope. The WFC is a prime focus optical mosaic camera with four EEV $2 \mathrm{k} \times 4 \mathrm{k}$ CCDs, yielding a collective field of view of $34^{\prime} \times 34^{\prime}$.

Galaxies within $\lesssim 1.5 \mathrm{Mpc}$ from the Local Group (LG) barycenter are defined as LG members. LGC will target 26 galaxies of the 35 most probable LG members based upon their visibility from La Palma. Our LG census will search for all strong emission-line populations, e.g., Wolf-Rayet's, luminous blue variables, planetary nebulae (Magrini et al. 2002), and $\mathrm{H}$ II regions.

IC-10 was selected as a test case for massive stars, as it is one of the most active star forming galaxies in the LG. Hodge \& Lee (1990) identified $144 \mathrm{H}$ II regions, which later led Massey et al. (1992) to probe the rich massive star population. A survey of WR stars (Massey \& Armandroff 1995) showed that IC-10 has twice the galaxy wide surface density of massive stars than any other LG galaxy.

Crowded field stellar photometry was performed with Stetson's (2001) version of DAOPHOT-II (Stetson 1987), ALLSTAR, ALLFRAME (Stetson 1994), and DAOGROW (Stetson 1990) packages. Instrumental magnitudes are presented, since IC-10 observations were taken under non-photometric conditions. Magnitudes shown were semi-calibrated by comparing our CMDs with previously 

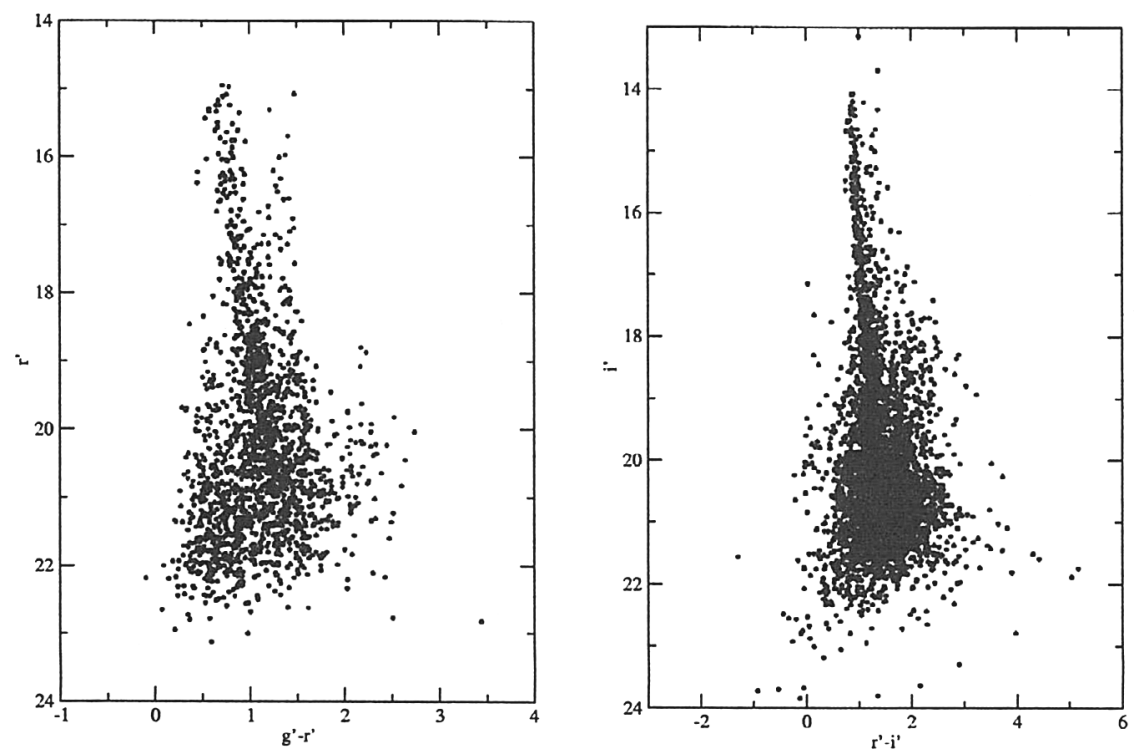

Figure 1. CMDs of the central 47 arcminutes ${ }^{2}$ of IC-10. (Left): $g^{\prime}-r^{\prime} v s . r^{\prime}$. (Right): $r^{\prime}-i^{\prime}$ vs. $i^{\prime}$.

published CMDs (Sakai et al. 1999; Massey \& Armandroff 1995), taking account of transformations between differing filter systems (Fukugita et al. 1996).

Astrometry on images will be performed with IRAF and wCS tools using the USNO-A2.0 catalogue. We will particularly be targeting OB stellar populations within IC-10, using a multi-object spectrograph (e.g., GMOS-N on Gemini). We have taken a series of photometric observations of open clusters with our $g^{\prime}, r^{\prime}, i^{\prime}$ filters to obtain a more accurate calibration of SLOAN spectral types. Procedures developed for this program will be easily generalized for crowded field photometry on other LG members.

\section{References}

Fukugita, M., Ichikawa, T., Gunn, J.E., et al. 1996, AJ 111, 1748

Garnett, D.R. 1990, ApJ 363,142

Hodge, P., Lee, M.G. 1990, PASP 102, 26

Magrini, L., Corradi, R.L.M., Walton, N.A., et al. 2002, A\&A 386, 869

Massey, P., Armandroff, T.E., Conti, P.S. 1992, AJ 103, 1159

Massey, P., Armandroff, T.E. 1995, AJ 109, 2470

Sakai, S., Madore, B.F., Freedman, W.L. 1999, ApJ 511, 671

Stetson, P.B. 1987, PASP 99, 191

Stetson, P.S. 1990, PASP 102, 932

Stetson, P.B. 1994, PASP 106, 250 